\title{
Design of Long Distance Network RFID Reader Based on ISO14443A
}

\author{
Yue $\mathrm{Wu}^{\mathrm{a}}$, Linli Zhou ${ }^{\mathrm{b}}$, Yue Duan $^{\mathrm{c}}$, Lei liu $^{\mathrm{c}}$ and Huifang Bao ${ }^{\mathrm{c}}$ \\ Institute of Intelligent Machines, Chinese Academy of Sciences, Hefei 230031, China. \\ awuyue@iim.ac.cn, ${ }^{\mathrm{b}}$ linlizhou@iim.ac.cn, ${ }^{\mathrm{c}}$ liulei12@mails.ucas.ac.cn
}

Keywords: RFID, ISO14443A, TMS320LF2812, Long distance, Network, RFID reader.

\begin{abstract}
This paper introduces the design of 13.56MHZ long distance RFID reader based on ISO14443A protocol. The hardware block diagram and the software flow chart of the reader are given. According to the experiment simulation, the sending signal and the receiving signal waveform are shown. The processor uses the TMS320LF2812 from Texas Instruments (TI). This design uses the analog circuit to build the RF circuit part of the reader to facilitate the amplification of power, increase the reading and writing distance. Through a lot of experiments, the actual reading distance of reader can reach $48 \mathrm{~cm}$, which supports MODBUS TCP/RTU Industrial Ethernet protocol and meets the requirements of industrial production. In addition, this design has been applied to the quality traceability of high-end wine, and will be extended to other industries.
\end{abstract}

\section{Introduction}

RFID technology has been considered to be one of the most promising technology in the 21 century. With the rapid development of the Internet of things, research on the key technology of RFID has become the key factor of promoting the construction of the Internet of things ${ }^{[1]}$. So far, the RF protocols working in $13.56 \mathrm{MHz}$ band mainly have ISO15693, ISO10536 and ISO14443A/B protocol. One of the most widely used is the ISO14443A protocol. The card supporting ISO14443A protocol is one of the most common used RF card worldwide with cheaper price than ISO15693 card. And each of its internal data area has a separate control double password area with high degree of confidentiality. In addition, the ISO14443A protocol has been written to the NFC standard. Since the launch of the first NOKIA NFC mobile phone, the major manufacturers have flocked to participate in the NFC mobile force. Domestic ZTE, HUAWEI also have launched its own NFC phone, and security traceability has become a typical application of NFC. Therefore, ISO14443A becomes the NFC standard communication protocol. But the mass production of the production line has a high requirement on the distance of the reader, and the normal distance of the ISO14443A protocol: $d \leq 10 \mathrm{~cm}$. On the other hand, the design and implementation of the network module are mostly not considered in the existing short distance and small power reading and writing. As a result, this design will combine the MODBUS TCP/RTU Industrial Ethernet protocol with the long distance and high power reader in order to cope with a variety of industrial field. To sum up, the development of long distance RFID reader based on ISO14443A protocol is of great significance.

\section{Hardware Design}

In this design, the core device of reader uses the DSPTMS320LF2812, a 32 bit fixed-point DSP chip launched by TI, which clock reached $150 \mathrm{MHz}$ with FLASH $128 \mathrm{~KB}$, RAM 18KB storage space. 
TMS320LF2812 internal integrates a 16-channel high-speed ADC with 12 bit, the external circuit uses analog circuits to build. As a result, the overall hardware structure is simple and clear.

The hardware structure of the reader is shown in Fig.1. The whole system is based on TMS320LF2812 as the core DSP system, not only through the configuration of R232, RS485 and USB to achieve two-way communication between PC and tag, but also be configured as a server or client and other hosts on the network for data transmission. The main task of DSP is to encode and decode the communication data.

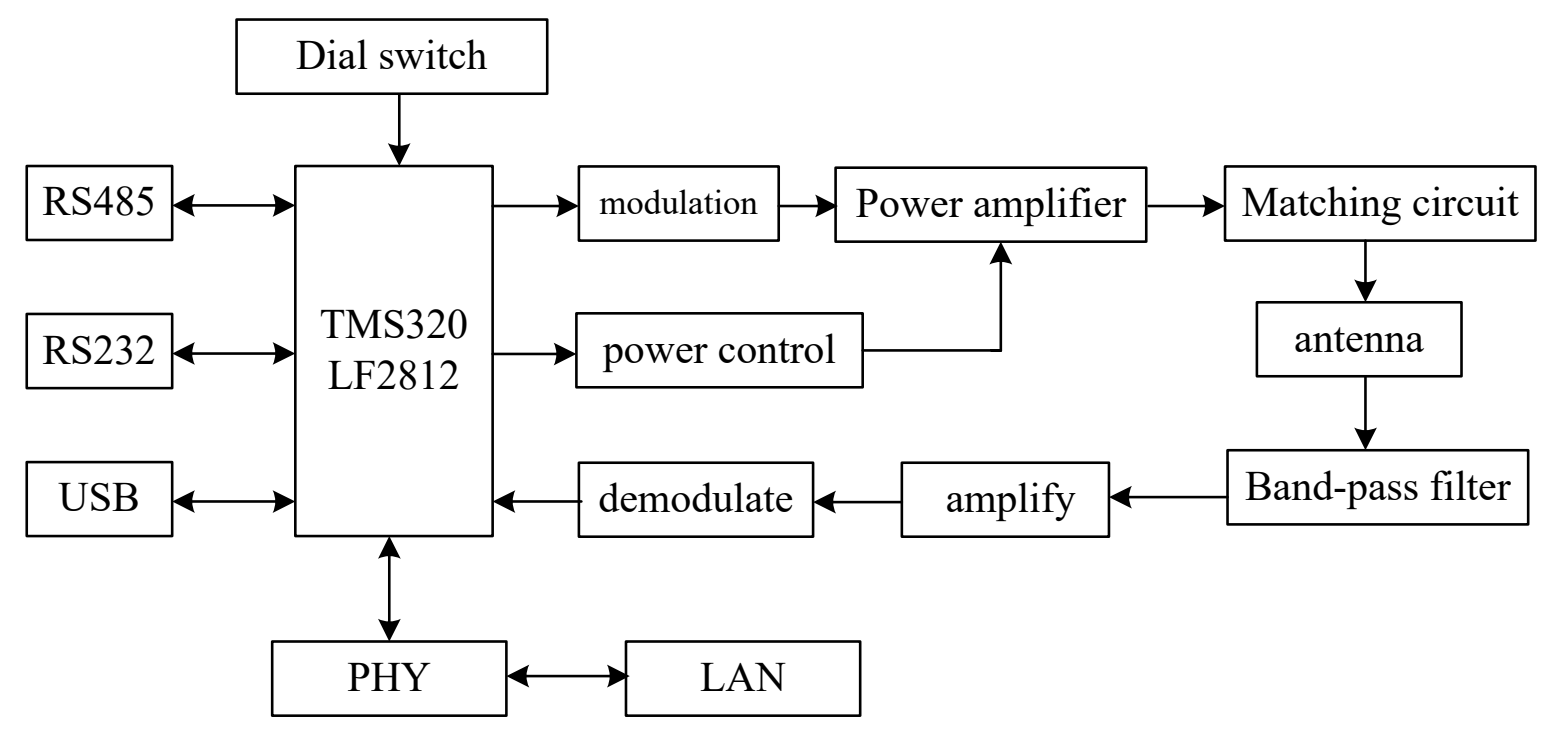

Fig. 1 Hardware block diagram of the reader

Control the DSP IO port to output $2.36 \mu \mathrm{s}\left(1 \mathrm{etc}=9.44 \mu \mathrm{s}, 2.36 \mu \mathrm{s}=\frac{1}{4} e t u\right)$ negative pulse signal to use pulse position modulation in local $13.56 \mathrm{MHz}$ carrier. As the modulation signal power weak lead to low RF signal: $V_{p p}<2 V$, so the modulation signal must be amplified at first, amplified RF signal: $V_{p p} \approx 50 \mathrm{~V}$, and then filtered and tuned to load on the antenna. Power amplifier circuit use NPN type radio-frequency power transistor MF426. The transmitting power was $4 \mathrm{~W}$ and the actual operating frequency up to $25 \mathrm{MHz}$. Through the potentiometer it can adjust power from $0.6 \mathrm{~W}$ to $6 \mathrm{~W}$. The final input impedance of the system is $50 \Omega$, the antenna coil in the working frequency of $13.56 \mathrm{MHz}$ showed impedance $Z$. In order to realize the matching with the power of $50 \Omega$ system $^{[2]}$, the system impedance is converted to $50 \Omega$ through passive matching circuit. And then the power from the reader terminal transmitted to the antenna matching circuit via coaxial cable $50 \Omega$.

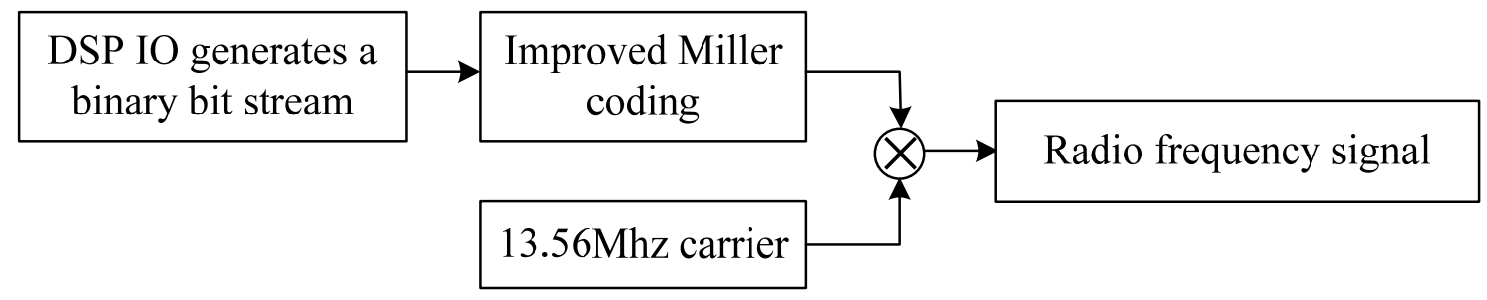

Fig. 2 Reader send data

In the ISO14443A protocol, the data transmission mode from lable to reader uses load modulation. The modulation circuit is shown in Figure 3. 


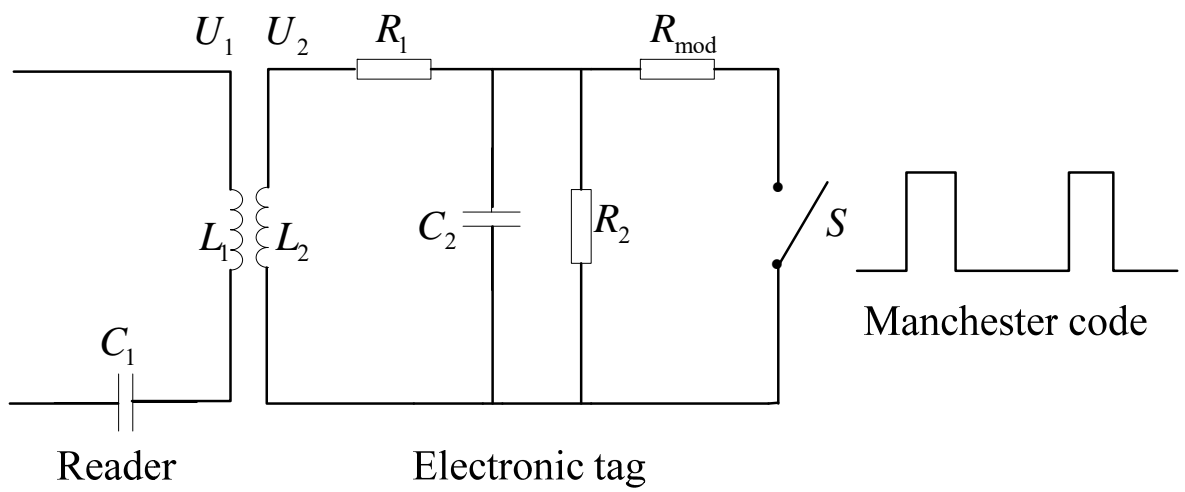

Fig. 3 Load modulation circuit

Binary data is encoding for the Manchester code. The $13.56 \mathrm{MHz}$ frequency is divided into $847 \mathrm{KHz}$ sub carrier, which is modulated by Manchester code ${ }^{[3]}$. When the code is " 1 ", the switch $\mathrm{S}$ is switched on, and the electronic tag load resistance $R_{\text {mod }}$ and $R_{2}$ are connected in parallel. When the code is " 2 ", the switch $\mathrm{S}$ is disconnected, the load resistance is $R_{2}$. With the parallel resonant load resistance decreased, the quality factor Q and the voltage $U_{2}$ at both ends of the resonant circuit are reduced. Because of the coil inductance coupling, the change of $U_{2}$ is passed to reader, the amplitude variation of voltage $U_{1}$ at both ends of the reader coil generates an amplitude modulation for the reader voltage, and the tag data is sent out as shown in Figure 4.

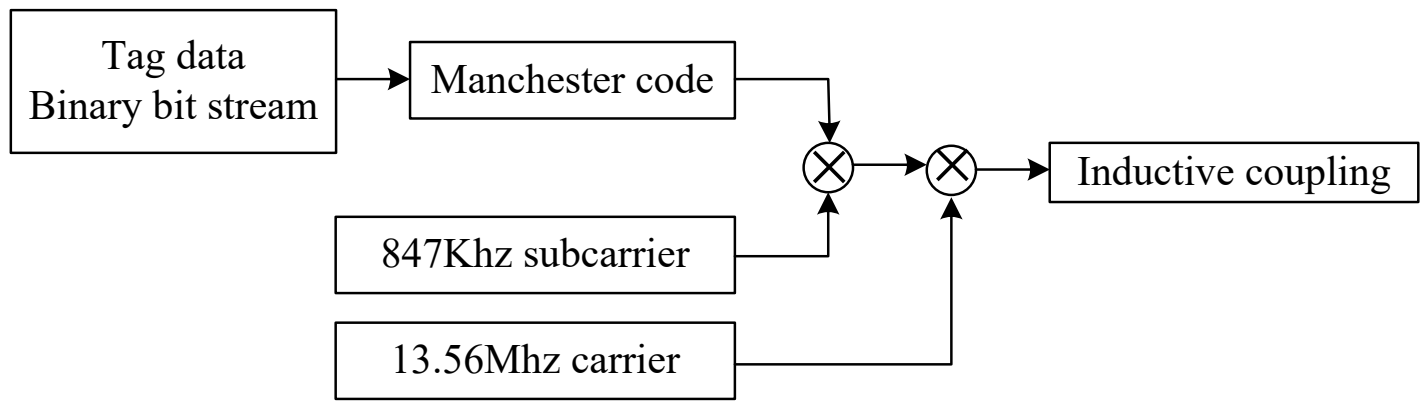

Fig. 4 Tag sending data

When the reader receives RF signals from the tag, it extracts the useful signal through the band-pass filter, then amplifies it and sends it to the demodulator. The demodulator mixes the sideband signal with the local $13.56 \mathrm{MHz}$ carrier, and finally filters ASK envelope detection. The resulting analog signal is send to the DSP's ADC channel for decoding, in order to get the label data as shown in Figure 5.

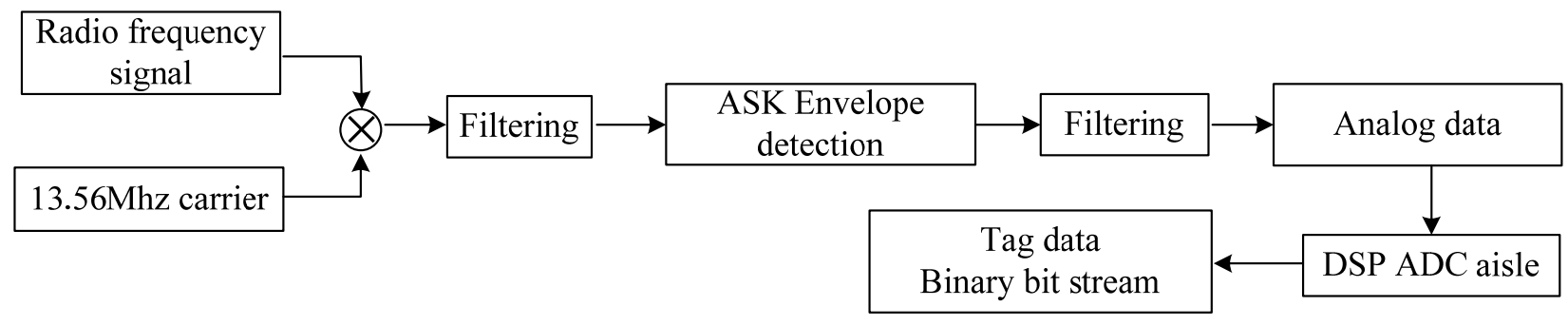

Fig. 5 The reader receives data

The DSPTMS320LF2812 transmits Modbus TCP data to the Ethernet bus via the PHY and transmits Modbus RTU data to the RS232 or RS485 bus. Considering the industrial field applications, serial interface and LAN interfaces need to be isolated in order to ensure reliable and stable electrical, support ISP download and be easy to module production. The main function of the dialing configuration is to configure the working mode, IP address and baud rate. 
The reader can be configured in a variety of operating modes. The client and server modes can be selected by the dialing configuration. The host on the network first establishe the connection with the reader via Ethernet and then communciates with reader according to the standard industrial Modbus protocol.

For PCB layout, accuracy of $1 \%$ resistor and $100 \mathrm{nF}$ decoupling capacitor should be placed close to the PHY device and connect to the power through the shortest path. The two pairs of differential signals (TD and RD) should be routed in parallel, avoid stubs and ensure length matching to away from common mode noise and EMI emissions. Ideally, there should be no crossovers or holes on the signal lines, and the differential lines should be as close to each other as possible. Reader Modbus operating mode shown in Figure 6.

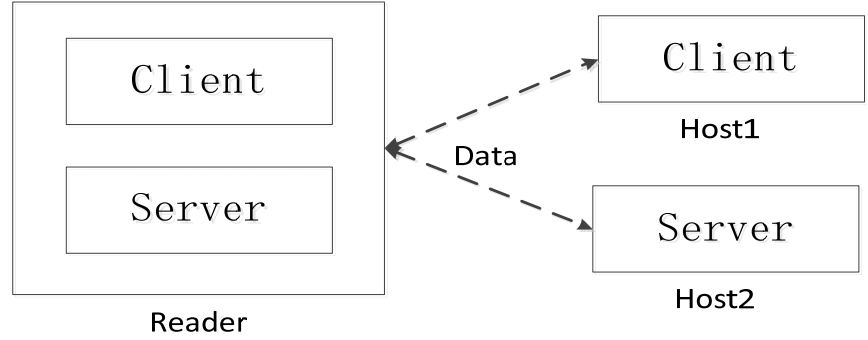

Fig. 6 Modbus operating mode diagram

\section{Software Design}

The reader software conforms to the ISO14443A protocol specification. As shown in Figure 7, the software is divided into the initialization program and the main loop program. The initialization program includes: initialize the system clock, initialize the system peripherals and initialize ISO14443A system. The main loop program includes serial tasks, USB tasks and reader tasks. After the reader power on, the reader's task is to send ISO14443A every 20ms REQA command frame, polling the label in the workplace. If there is a label in the workplace, the reader will get the label UID (Unique-Identification) ${ }^{[4,5,6,7]}$ and save it in the circular queue. If the work mode is HTLA mode, the reader will immediately hang this label. The reader will parse the command frame and execute the corresponding instruction when receiving the serial or USB command frame. After the reader completes the command, it will save the processing result to the response frame, send it to the PC through the serial port and finish the sending and receiving process of the whole system ${ }^{[8]}$. 


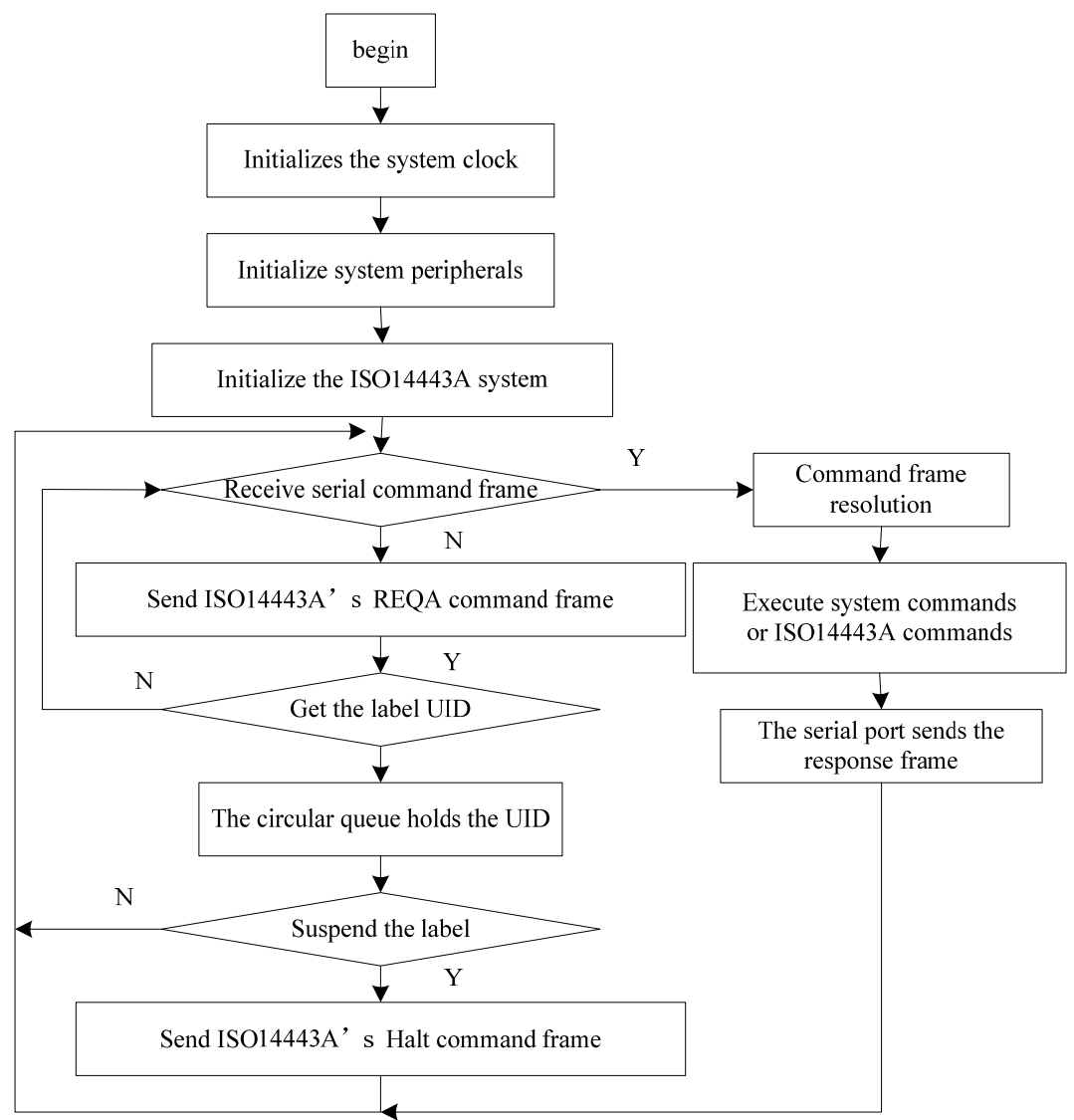

Fig. 7 The Communication Flow Chart of Reader and ISO14443A Card

The process of establishing a connection protocol between reader and the network host requires a three-way handshake. The client sends a TCP packet with the SYN flag to the server. The server responds to the client is the second packet in the three-way handshake with the ACK flag and the SYN flag. Therefore, it indicates the response to the SYN message of the client. It also signals the SYN to the client and inquires whether the client is ready for data communication. The client must respond an ACK packet to the service segment again. Because TCP connections are full duplex, each direction must be turned off individually. The principle is that when a party completes its task of sending data, it can send a FIN to terminate the connection in that direction. Receiving a FIN only means that there is no data flow in that direction, but a TCP connection can still send data after receiving a FIN. The one that closes first will perform an active shutdown while the other will perform a passive shutdown. The TCP state transition diagram of reader shown in Figure 8. 


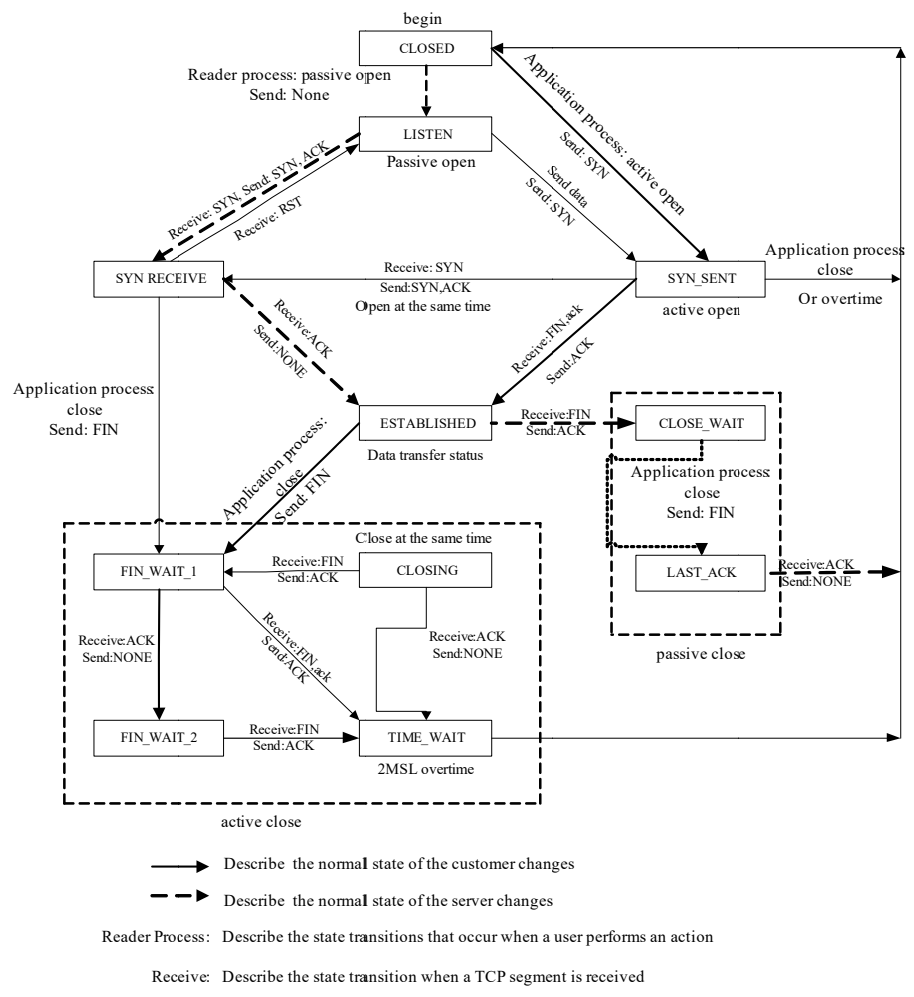

Fig. 8 The TCP State Transition Diagram of the Reader

\section{Experimental Results and Analysis}

DSP modulation $13.56 \mathrm{MHz}$ carrier to obtain the RF signal by generating a negative pulse signal. The $\mathrm{RF}$ signal shown on the oscilloscope shown in Figure 9. The RF signal $V_{p p}<2 V$, it is too weak to read the long-range label.

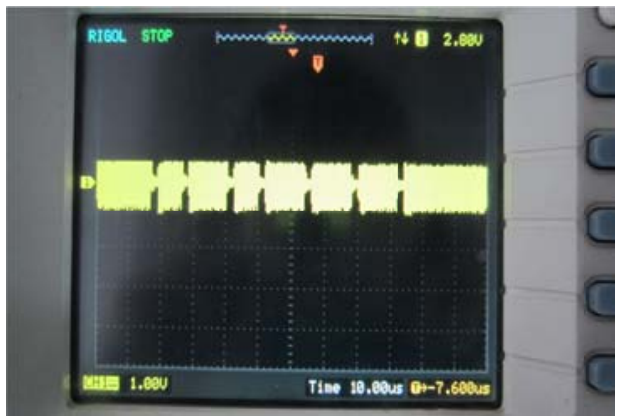

Fig. 9 RF signal before power amplification

The signal peak rises to $50 \mathrm{~V}$ through the power amplifier circuit, as shown in Figure 10 . The negative pulse of the signal is interpreted as a REQA command with a value of "26" in ISO14443A, which is sent once every $20 \mathrm{~ms}$ to poll the labels in the workplace.

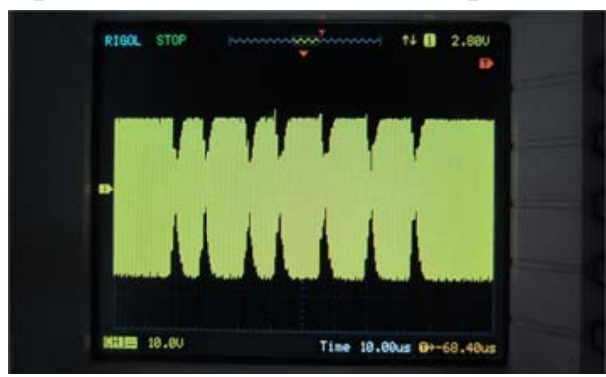

Fig. 10 The radio frequency signal that the reader sends 
After receiving RF signals from the tag, the reader obtains the analog signal of the tag data through filtering, demodulation and detection. The analog signal data is shown in Fig. 11. The analog signal will be sent to the DSP's ADC channel and become the label data after sampling and the final decision.

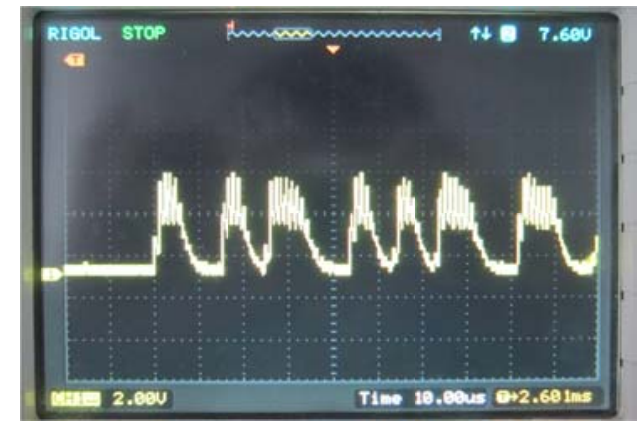

Fig. 11 Analog data waiting in the DSP ADC channel

\section{Summary}

Based on the ISO14443A protocol, this paper designs and realizes the long distance RFID reader working in the frequency band of $13.56 \mathrm{MHz}$. The antenna size is $30 \mathrm{~cm} \times 40 \mathrm{~cm}^{[9]}$ and the maximum reading and writing distance can reach $48 \mathrm{~cm}$. The reader can realize the readout of ISO14443A card UID, the read and write of single tag data block and the operation of value segment.

The design can be applied in the factory production line to achieve mass production with the NFC anti-counterfeiting traceability software in mobile phone. Consumers can effectively identify the authenticity and related information of the product. This design has been applied to the quality traceability of high-end wine, and will be extended to other industries to make up the related quality traceability system.

\section{Acknowledgement}

This research is financially supported by Key Projects in the National Science\& Technology Pillar Program in the Twelve Five-year Plan Period (No: 2015BAD18B01).

\section{References}

[1] Se Won Oh, Hyochan Bang, Jae Gak Hwang, Light-weight RFID device interface for controlling RFID tag memory access, J. Advanced Communication Technology (ICACT), 2010.

[2] Joshua D. Griffin, Andreas Haldi, Bernard Kippelenetc, RF Tag Antenna Performance on Various Materials Using Radio Link Budgets, 2006.

[3] Colin Warwick, Mike Mulligan, Using Behavioral Models to Drive RF Design and Verify System Performance, 2005.

[4] ISO COMMITTEE DRAFT 14443.Identification cards-Contactless integrated circuit cards-Vicinity cards.

[5]Stmicroelectronics.STM31F105xxSTM32F107xx[EB/OL].2011.8.http://www.stmicroelectronics .cinternet/com/TECHNICAL_RESOURCES/TECHNICAL_LITERATURE/DATASHEET/CD002 20364. 